Website: http://revistas.lamolina.edu.pe/index.php/acu/index

(C) Universidad Nacional Agraria La Molina, Lima - Perú

\title{
La innovación educativa y su relación con el rendimiento académico de los estudiantes del curso de economía general de la Universidad Nacional Agraria La Molina
}

\author{
The educational innovation and its relation with the academic performance of the stude
}

\author{
Gorki Humberto Llerena Lazo de la Vega ${ }^{1}$
}

\begin{abstract}
Resumen
La importancia de la investigación a través de la innovación educativa busca promover el cambio y las transformaciones en el aspecto educativo en la Universidad Nacional Agraria La Molina (UNALM), mediante la innovación educativa se busca promover actitudes positivas hacia el cambio y estimular la investigación en los estudiantes mediante las innovaciones educativas beneficiando a la universidad y a la sociedad. Para ello, se planteó como objetivo conocer la relación que existe entre la innovación educativa con el rendimiento académico de los estudiantes del curso de Economía General de la UNALM. La metodología involucra un estudio descriptivo-explicativo, con un diseño descriptivo correlacional. Los resultados del presente estudio coinciden con la necesidad de innovar en la educación universitaria con un efectivo proceso de cambios sociales y educacionales. Los resultados indican que los cambios que se están produciendo en la sociedad afectan a la educación y al trabajo del docente. Una sociedad del conocimiento requiere de instituciones educativas orientadas hacia la innovación y la calidad. En conclusión la innovación educativa se relaciona significativamente con el rendimiento académico, la innovación curricular, la innovación científica y tecnológica; y la innovación en la educación superior permiten innovar en la educación universitaria; así mismo, permite mejorar el rendimiento académico de los estudiantes en el ámbito personal, cognitivo, motivacional, afectivo y familiar. El coeficiente de correlación de $r$ Pearson es de 0,45 a un nivel de significancia de 0,05 .
\end{abstract}

Palabras clave: Innovación educativa; innovación social; innovación curricular; rendimiento académico; ámbito personal; ámbito cognitivo; ámbito motivacional; ámbito afectivo; ámbito familiar.

\begin{abstract}
The importance of research is Through educational innovation seeks to promote change and transformation in the educational aspect at the Universidad Nacional Agraria La Molina (UNALM), through educational innovations seeks to promote positive attitudes towards change and stimulate research in students through educational innovations benefiting the university and society. To this end, we propose as a goal: To know the relationship that exists between educational innovation and the academic performance of the students of the General Economics Course of theUNALM. The methodology involves a descriptive-explanatory study, with a descriptive correlational design. The results of this study coincide with the need to innovate in university education with an effective process of social and educational changes. The results indicate that the changes that are taking place in our society affect the education and work of the teacher. A knowledge society requires educational institutions oriented towards innovation and quality. The conclusion were: Educational innovation is significantly related to academic performance, since social innovation, strategic educational innovation, curricular innovation, scientific and technological innovation and innovation in higher education allow innovation in university education; likewise, it allows improving the academic performance of students in the personal, cognitive, motivational, affective and family spheres. The correlation coefficient of $r$ Pearson is 0,45 at a significance level of 0,05 .
\end{abstract}

Keywords: Educational innovation; social innovation; curricular innovation; academic performance; personal scope; cognitive field; motivational field; affective field;family environment.

\section{Introducción}

El estudio se justifica por la relevancia social del tema y sobre el manejo de la variable innovación educativa en la educación universitaria el cual es escaso en nuestro medio académico e intelectual. La importancia de la investigación es: A través de la innovación educativa se busca promover el cambio y las transformaciones en el aspecto educativo en la UNALM, mediante las innovaciones educativas busca promover actitudes positivas hacia el cambio y estimular la investigación en los estudiantes mediante las innovaciones educativas beneficiando a la universidad y a la sociedad. En cuanto al alcance de la investigación son los estudiantes del curso de economía general del semestre académico 2012 de la Facultad de Economía y Planificación de la UNALM.

\section{Materiales y métodos}

La investigación se realizó mediante un diseño descriptivo correlacional con un grupo único. Se seleccionó 62

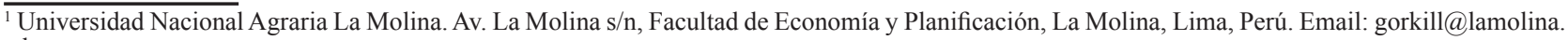
edu.pe
} 
estudiantes del curso de Economía General. El método involucró la elaboración de dos cuestionarios para evaluar las correlaciones de las variables de estudio, que fue validado por expertos y se sometieron a la prueba de confiabilidad de alfa de Cronbach, las puntuaciones obtenidas fue de 0,79 y 0,71 para ambos cuestionarios. El análisis de los datos se realizó mediante estadísticos descriptivos de frecuencias y de tendencia central y de variabilidad (media, desviación estándar, moda y rangos), se realizó la prueba de normalidad de Kolmogorov-Smirnov y para la comprobación de las hipótesis se recurrió a la prueba "r de Pearson" de muestra única, con la finalidad de comprobar la correlación de las variables.

\section{Resultados y discusión}

Los resultados del presente estudio coinciden con la necesidad de innovar en la educación universitaria con un efectivo proceso de cambios sociales y educacionales.

Después de la recopilación de datos de los 62 estudiantes del curso de Economía General de la UNALM del año académico 2012, con el cuestionario A y B, se realizó el análisis estadístico y luego se presenta los resultados a través de tablas y gráficos.

Tabla 1. Distribución de frecuencias de la innovación educativa

\begin{tabular}{|c|c|c|c|c|c|}
\hline \multicolumn{6}{|c|}{ La innovación educativa } \\
\hline & & Frecuencia & $\%$ & \% válido & $\%$ acumulado \\
\hline \multirow{30}{*}{ Válidos } & 81 & 3 & 4,8 & 4,8 & 4,8 \\
\hline & 85 & 1 & 1,6 & 1,6 & 6,5 \\
\hline & 86 & 1 & 1,6 & 1,6 & 8,1 \\
\hline & 87 & 1 & 1,6 & 1,6 & 9,7 \\
\hline & 88 & 2 & 3,2 & 3,2 & 12,9 \\
\hline & 89 & 1 & 1,6 & 1,6 & 14,5 \\
\hline & 90 & 3 & 4,8 & 4,8 & 19,4 \\
\hline & 91 & 3 & 4,8 & 4,8 & 24,2 \\
\hline & 93 & 3 & 4,8 & 4,8 & 29,0 \\
\hline & 94 & 1 & 1,6 & 1,6 & 30,6 \\
\hline & 95 & 2 & 3,2 & 3,2 & 33,9 \\
\hline & 96 & 3 & 4,8 & 4,8 & 38,7 \\
\hline & 97 & 1 & 1,6 & 1,6 & 40,3 \\
\hline & 98 & 1 & 1,6 & 1,6 & 41,9 \\
\hline & 99 & 6 & 9,7 & 9,7 & 51,6 \\
\hline & 100 & 5 & 8,1 & 8,1 & 59,7 \\
\hline & 101 & 3 & 4,8 & 4,8 & 64,5 \\
\hline & 102 & 4 & 6,5 & 6,5 & 71,0 \\
\hline & 103 & 4 & 6,5 & 6,5 & 77,4 \\
\hline & 104 & 1 & 1,6 & 1,6 & 79,0 \\
\hline & 105 & 3 & 4,8 & 4,8 & 83,9 \\
\hline & 107 & 1 & 1,6 & 1,6 & 85,5 \\
\hline & 108 & 1 & 1,6 & 1,6 & 87,1 \\
\hline & 110 & 1 & 1,6 & 1,6 & 88,7 \\
\hline & 111 & 1 & 1,6 & 1,6 & 90,3 \\
\hline & 112 & 1 & 1,6 & 1,6 & 91,9 \\
\hline & 113 & 2 & 3,2 & 3,2 & 95,2 \\
\hline & 114 & 2 & 3,2 & 3,2 & 98,4 \\
\hline & 118 & 1 & 1,6 & 1,6 & 100,0 \\
\hline & Total & 62 & 100,0 & 100,0 & \\
\hline
\end{tabular}

Al observar la distribución de frecuencias que contiene la Tabla 1 de las puntuaciones del cuestionario A (La innovación educativa) se advierte que fluctúan entre 81 y 118; y las puntuaciones muestran una tendencia a acumularse ligeramente en la parte inferior de la distribución.

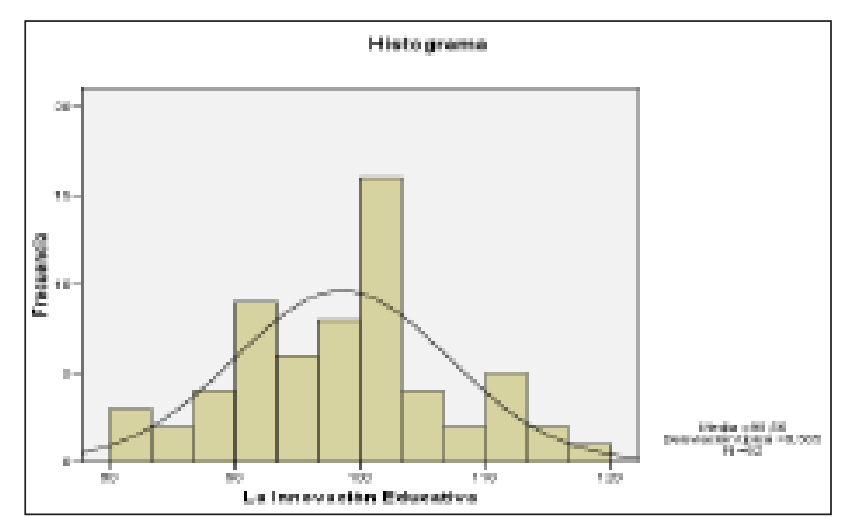

Figura 1. Histograma de la innovación educativa

Tabla 2. Distribución de frecuencias del rendimiento académico

\begin{tabular}{|c|c|c|c|c|c|}
\hline & & \multicolumn{3}{|c|}{ El rendimiento académico } & \multirow[b]{2}{*}{ \%acumulado } \\
\hline & & Frecuencia & $\%$ & \% válido & \\
\hline & 78 & 1 & 1,6 & 1,6 & 1,6 \\
\hline & 80 & 1 & 1,6 & 1,6 & 3,2 \\
\hline & 82 & 1 & 1,6 & 1,6 & 4,8 \\
\hline & 84 & 1 & 1,6 & 1,6 & 6,5 \\
\hline & 85 & 1 & 1,6 & 1,6 & 8,1 \\
\hline & 86 & 2 & 3,2 & 3,2 & 11,3 \\
\hline & 87 & 2 & 3,2 & 3,2 & 14,5 \\
\hline & 88 & 2 & 3,2 & 3,2 & 17,7 \\
\hline & 89 & 3 & 4,8 & 4,8 & 22,6 \\
\hline & 90 & 1 & 1,6 & 1,6 & 24,2 \\
\hline & 91 & 2 & 3,2 & 3,2 & 27,4 \\
\hline & 92 & 2 & 3,2 & 3,2 & 30,6 \\
\hline & 93 & 1 & 1,6 & 1,6 & 32,3 \\
\hline & 94 & 1 & 1,6 & 1,6 & 33,9 \\
\hline & 95 & 3 & 4,8 & 4,8 & 38,7 \\
\hline Válidos & 96 & 1 & 1,6 & 1,6 & 40,3 \\
\hline & 97 & 4 & 6,5 & 6,5 & 46,8 \\
\hline & 98 & 6 & 9,7 & 9,7 & 56,5 \\
\hline & 99 & 1 & 1,6 & 1,6 & 58,1 \\
\hline & 100 & 1 & 1,6 & 1,6 & 59,7 \\
\hline & 101 & 6 & 9,7 & 9,7 & 69,4 \\
\hline & 102 & 3 & 4,8 & 4,8 & 74,2 \\
\hline & 103 & 4 & 6,5 & 6,5 & 80,6 \\
\hline & 104 & 3 & 4,8 & 4,8 & 85,5 \\
\hline & 105 & 2 & 3,2 & 3,2 & 88,7 \\
\hline & 106 & 1 & 1,6 & 1,6 & 90,3 \\
\hline & 107 & 2 & 3,2 & 3,2 & 93,5 \\
\hline & 109 & 2 & 3,2 & 3,2 & 96,8 \\
\hline & 110 & 1 & 1,6 & 1,6 & 98,4 \\
\hline & 116 & 1 & 1,6 & 1,6 & 100,0 \\
\hline & Total & 62 & 100,0 & 100,0 & \\
\hline
\end{tabular}


La distribución de frecuencias del cuestionario A ( La innovación educativa) está representado gráficamente por el histograma con la curva normal, donde se identifica la media, la desviación típica y la muestra.

Al observar la distribución de frecuencias que contiene la Tabla 2 de las puntuaciones del cuestionario B (El rendimiento académico) se advierte que fluctúan entre 78 y 116; y que las puntuaciones muestran una tendencia a acumularse ligeramente en la parte inferior de la distribución.

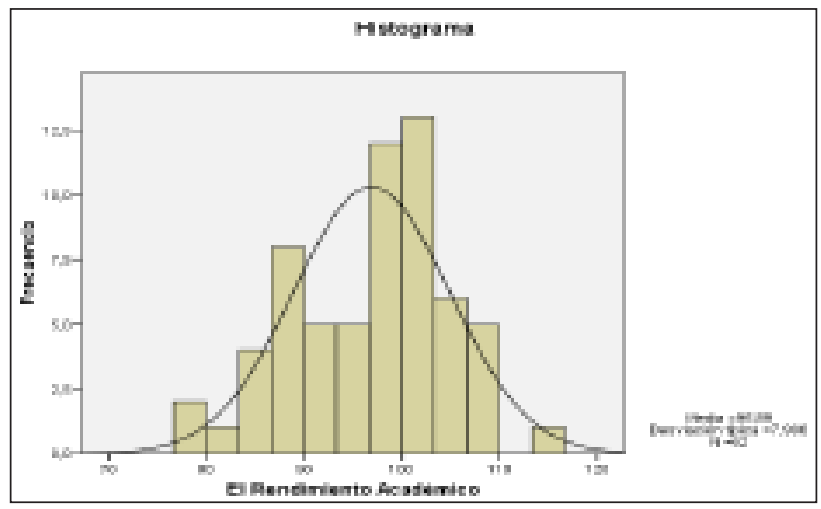

Figura 2. Histograma del rendimiento académico

La distribución de frecuencias del cuestionario B (El rendimiento académico) está representado gráficamente por el histograma, con la curva normal, donde se identifica la media, la desviación típica y la muestra.

\section{Estadísticos descriptivos}

Tabla 3. Medidas de centralidad y de variabilidad de la innovación educativa

\begin{tabular}{ccc}
\hline \multicolumn{3}{c}{ Estadísticos } \\
La innovación educativa \\
\hline \multirow{2}{*}{ Válidos } & 62 \\
& Perdidos & 0 \\
& Media & 98,56 \\
Error típ. de la media & 1,088 \\
Mediana & 99,00 \\
Moda & 99 \\
Desv. típ. & 8,565 \\
Varianza & 73,365 \\
Rango & 37 \\
Mínimo & 81 \\
Máximo & 118 \\
\hline
\end{tabular}

Cabe destacar que, al describir los datos de las puntuaciones del cuestionario A de la innovación educativa, se interpreta que las medidas de tendencia central y de la variabilidad en conjunto, no aisladamente. Se tomó en cuenta todas las medidas. La puntuación que más se repitió fue 99 . El 50\% de los estudiantes está por encima de 99 y el restante $50 \%$ se sitúa por debajo de este valor. En promedio, los estudiantes se ubican en 98,56 La máxima puntuación que se obtuvo fue 118. Las puntuaciones de los estudiantes tienden a ubicarse en valores por debajo de 99 .

Al describir los datos de las puntuaciones del cuestionario B del rendimiento académico, se interpretan las medidas de tendencia central y de la variabilidad en conjunto, no aisladamente. Se tomó en cuenta todas las medidas. La puntuación que más se repitió fue 98. El $50 \%$ de los estudiantes está por encima de 98 y el restante $50 \%$ se sitúa por debajo de este valor. En promedio, los estudiantes se ubican en 96,89 La máxima puntuación que se obtuvo fue 116. Las puntuaciones de los estudiantes tienden a ubicarse en valores por debajo de 98 .

Tabla 4. Medidas de centralidad y de variabilidad del rendimiento académico

\begin{tabular}{lrr}
\hline \multicolumn{2}{c}{ Estadísticos } \\
\hline $\mathrm{N}$ & El rendimiento académico \\
\hline Media & Válidos & 62 \\
Error típ. de la media & 0 \\
Mediana & 96,89 \\
Moda & 1,016 \\
Desv. típ. & 98,00 \\
Varianza & $98^{\mathrm{a}}$ \\
Rango & 7,996 \\
Mínimo & 63,938 \\
Máximo & 38 \\
\hline
\end{tabular}

a. Existen varias modas. Se mostrará el menor de los valores.

Descripción de las variables.

Variable X: La Innovación Educativa

Variable Y: El Rendimiento Académico

Descripción de las cinco hipótesis específicas.

Coeficiente de correlación de Pearson $r$ :

Tabla 5. Coeficiente de correlación de Pearson de las dimensiones la innovación social y el ámbito personal

\begin{tabular}{|c|c|c|c|}
\hline \multicolumn{4}{|c|}{ Correlaciones } \\
\hline & & $\begin{array}{c}\mathrm{La} \\
\text { innovación } \\
\text { social }\end{array}$ & $\begin{array}{l}\text { El ámbito } \\
\text { personal }\end{array}$ \\
\hline \multirow{3}{*}{$\begin{array}{l}\text { La } \\
\text { innovación } \\
\text { social }\end{array}$} & $\begin{array}{l}\text { Correlación de } \\
\text { Pearson }\end{array}$ & 1 &, 083 \\
\hline & Sig. (bilateral) & & ,522 \\
\hline & $\mathrm{N}$ & 62 & 62 \\
\hline \multirow{3}{*}{$\begin{array}{l}\text { El ámbito } \\
\text { personal }\end{array}$} & $\begin{array}{l}\text { Correlación de } \\
\text { Pearson }\end{array}$ & ,083 & 1 \\
\hline & Sig. (bilateral) & ,522 & \\
\hline & $\mathrm{N}$ & 62 & 62 \\
\hline
\end{tabular}

En el diagrama de dispersión se puede visualizar gráficamente una correlación positiva muy débil entre estas dimensiones. 


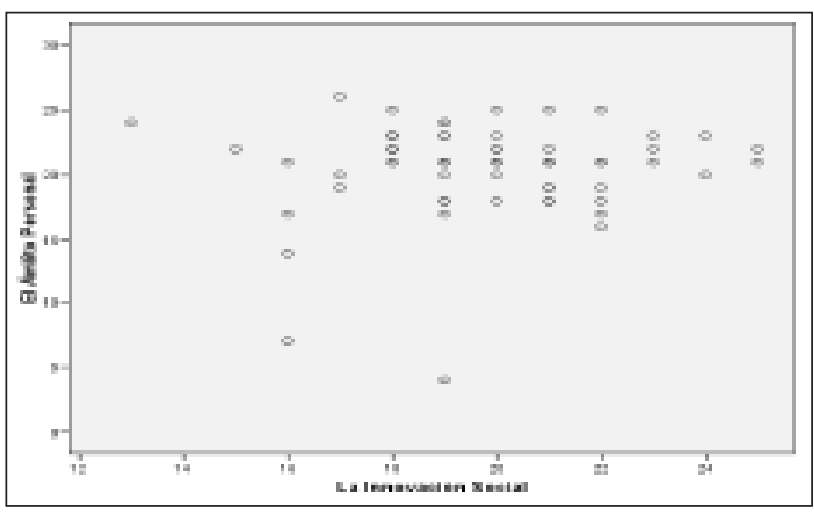

Figura 3. Diagrama de dispersión de las dimensiones la innovación social

Tabla 6. Coeficiente de correlación de Pearson de las dimensiones la innovación educativa estratégica y el ámbito cognitivo

\begin{tabular}{llrr}
\hline \multicolumn{4}{c}{ Correlaciones } \\
\hline & \multicolumn{1}{c}{$\begin{array}{c}\text { La } \\
\text { innovación } \\
\text { educativa } \\
\text { estratégica }\end{array}$} & $\begin{array}{r}\text { El ámbito } \\
\text { cognitivo }\end{array}$ \\
\hline $\begin{array}{llrr}\text { La innovación } \\
\text { educativa } \\
\text { estratégica }\end{array}$ & $\begin{array}{l}\text { Correlación } \\
\text { de Pearson }\end{array}$ & 1 &, 182 \\
& Sig. (bilateral) & &, 156 \\
& $\mathrm{~N}$ & 62 & 62 \\
$\begin{array}{l}\text { El ámbito } \\
\text { cognitivo }\end{array}$ & Correlación &, 182 & 1 \\
& Sig. (bilateral) &, 156 & \\
\hline & $\mathrm{N}$ & 62 & 62 \\
\hline
\end{tabular}

Se ha encontrado un coeficiente de correlación de Pearson entre la innovación educativa estratégica y el rendimiento académico: ámbito cognitivo igual a 0,18 ; lo que indica que existe una correlación positiva débil entre estas dimensiones.

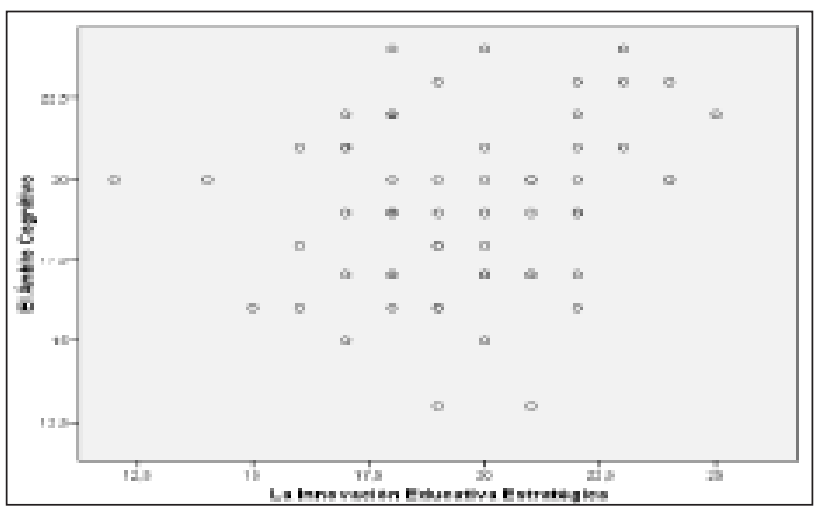

Figura 4. Diagrama de dispersión de las dimensiones la innovación educativa estratégica y el ámbito cognitivo

En el diagrama de dispersión se puede visualizar gráficamente una correlación positiva débil entre estas dimensiones.
Tabla 7. Coeficiente de correlación de Pearson de las dimensiones la innovación curricular y el ámbito motivacional

\begin{tabular}{llrr}
\hline \multicolumn{4}{c}{ Correlaciones } \\
\hline & $\begin{array}{c}\text { La } \\
\text { innovación } \\
\text { curricular }\end{array}$ & $\begin{array}{c}\text { El ámbito } \\
\text { motivacional }\end{array}$ \\
\hline La & Correlación de Pearson & 1 &, 137 \\
innovación & Sig. (bilateral) & &, 290 \\
curricular & $\mathrm{N}$ & 62 & 62 \\
& Correlación de Pearson &, 137 & 1 \\
El ámbito & Sig. (bilateral) &, 290 & \\
motivacional & $\mathrm{N}$ & 62 & 62 \\
\hline
\end{tabular}

Se ha encontrado un coeficiente de correlación de Pearson entre la innovación curricular y el rendimiento académico: ámbito motivacional igual a 0,14 ; lo que indica que existe una correlación positiva débil entre estas dimensiones.

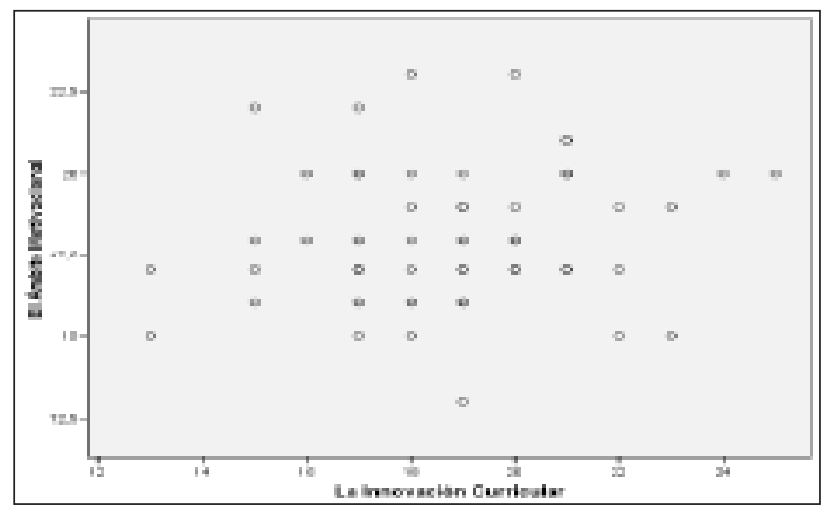

Figura 5. Diagrama de dispersión de las dimensiones la innovación curricular y el ámbito motivacional

En el diagrama de dispersión se puede visualizar gráficamente una correlación positiva débil entre estas dimensiones.

Tabla 8. Coeficiente de correlación de Pearson de las dimensiones la innovación científica y tecnológica y el ámbito afectivo

\begin{tabular}{llrr}
\hline \multicolumn{3}{c}{ Correlaciones } \\
\hline & $\begin{array}{c}\text { La } \\
\text { innovación } \\
\text { científica y } \\
\text { tecnológica }\end{array}$ & $\begin{array}{c}\text { El } \\
\text { afmbito } \\
\text { afectivo }\end{array}$ \\
\hline La & Correlación de Pearson & 1 &, 138 \\
innovación & Sig. (bilateral) & &, 286 \\
$\begin{array}{l}\text { científica y } \\
\text { tecnológica }\end{array}$ & $\mathrm{N}$ & 62 & 62 \\
El ámbito & Correlación de Pearson &, 138 & 1 \\
afectivo & Sig. (bilateral) &, 286 & \\
& $\mathrm{~N}$ & 62 & 62 \\
\hline
\end{tabular}

Se ha encontrado un coeficiente de correlación de Pearson entre la innovación científica y tecnológica y el 
rendimiento académico: ámbito afectivo igual a 0,14 ; lo que indica que existe una correlación positiva débil entre estas dimensiones.

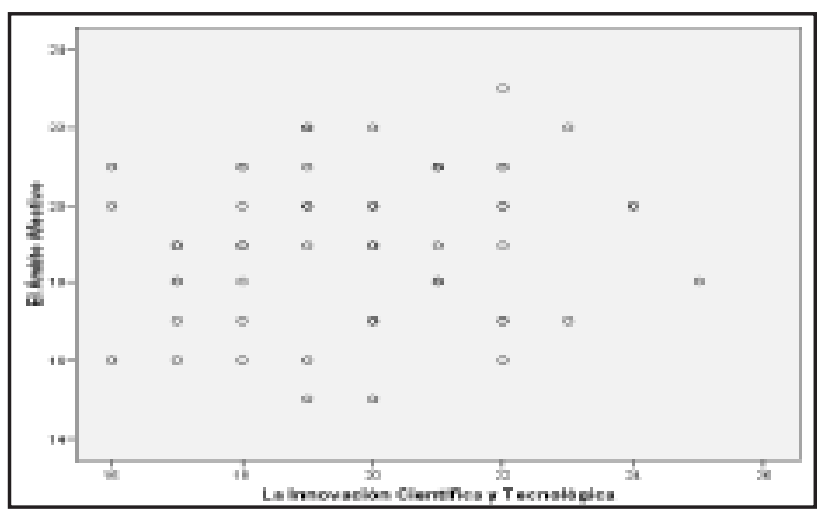

Figura 6. Diagrama de dispersión de las dimensiones la innovación científica y tecnológica y el ámbito afectivo

En el diagrama de dispersión se puede visualizar gráficamente una correlación positiva débil entre estas dimensiones.

Tabla 9. Coeficiente de correlación de Pearson de las dimensiones la innovación en la educación superior y el ámbito familiar

\begin{tabular}{llrr}
\hline \multicolumn{4}{c}{ Correlaciones } \\
& \multicolumn{1}{c}{$\begin{array}{c}\text { La innovación } \\
\text { en la educación } \\
\text { superior }\end{array}$} & $\begin{array}{c}\text { El } \\
\text { ámbito } \\
\text { familiar }\end{array}$ \\
\hline $\begin{array}{l}\text { La innovación } \\
\text { en la educación } \\
\text { superior }\end{array}$ & $\begin{array}{l}\text { Correlación de } \\
\text { Pearson }\end{array}$ & 1 &, $367^{* *}$ \\
& Sig. (bilateral) & &, 003 \\
& N & 62 & 62 \\
El ámbito & Correlación de &, $367^{* *}$ & 1 \\
familiar & Pearson & & \\
& Sig. (bilateral) &, 003 & \\
& $\mathrm{~N}$ & 62 & 62 \\
\hline
\end{tabular}

**. La correlación es significativa al nivel 0,01 (bilateral).

Se ha encontrado un coeficiente de correlación de Pearson entre la innovación en la educación superior y el rendimiento académico: ámbito familiar igual a 0,37 ; lo que indica que existe una correlación positiva débil entre estas dimensiones.

En el diagrama de dispersión se puede visualizar gráficamente una correlación positiva débil entre estas dimensiones.

Los resultados del presente estudio coinciden con la necesidad de innovar en la educación universitaria con un efectivo proceso de cambios sociales y educacionales. Los resultados a los que alude Marcelo et al. (2010), indican que los cambios que se están produciendo en la sociedad afectan a la educación y al trabajo del docente. Una sociedad del conocimiento requiere de instituciones educativas orientadas hacia la innovación y la calidad.
De 62 estudiantes del curso de Economía General de la UNALM, encuestados con el instrumento A (Ítem 25) sobre la Innovación Educativa, 37 de ellos están muy de acuerdo y totalmente de acuerdo en que en la sociedad del conocimiento resulta primordial la producción de conocimientos.

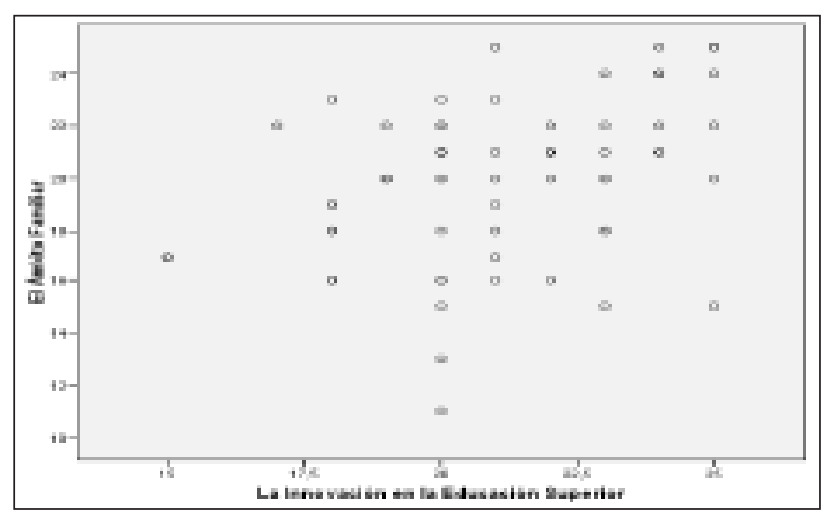

Figura 7. Diagrama de dispersión de las dimensiones la innovación en la educación superior y el ámbito familiar

Por otra parte, los resultados de la prueba de hipótesis específica 1 concuerdan con los que hace mención García (2004), en el sentido de que la innovación social y educativa es una construcción colectiva y que a su vez es un compromiso individual y social con la transformación de la realidad. De 62 estudiantes del curso de Economía General de la U, encuestados con el instrumento A (Ítem 9) sobre la Innovación Educativa, 52 de ellos están muy de acuerdo y totalmente de acuerdo en que en la participación de los ciudadanos en la innovación educativa es fundamental.

Así mismo, los resultados de la prueba de hipótesis 2 coinciden con las ideas puestas en de la investigación de Marcelo et al. (2010); coinciden con la idea que la mayoría de las instituciones educativas innovadoras que llevan a cabo proyectos de innovación son públicos y se reparten proporcionalmente la responsabilidad, además cuentan con un profesorado con experiencia ya que la mayoría suele tener una antigüedad de entre seis y 10 años y que se implica de forma activa en los procesos de planificación de los proyectos, dispuesto a asumir riesgos y a llevar a cabo nuevas iniciativas; y con un equipo directivo, donde están interesados en los proyectos de innovación. De 62 estudiantes del curso de economía general de la UNALM, encuestados con el instrumento A (Ítem 8) sobre la Innovación Educativa, 39 de ellos están muy de acuerdo y totalmente de acuerdo en que en las diferentes instituciones educativas los equipos directivos deben liderar las innovaciones educativas.

\section{Conclusiones}

La innovación educativa se relaciona significativamente con el rendimiento académico, ya que la innovación social, la innovación educativa estratégica, la innovación curricular, la innovación científica y tecnológica; y la 
innovación en la educación superior permiten innovar en la educación universitaria; así mismo, permite mejorar el rendimiento académico de los estudiantes en el ámbito personal, cognitivo, motivacional, afectivo y familiar. El coeficiente de correlación de $r$ Pearson es de 0,45 a un nivel de significancia de 0,05 .

La innovación en la educación superior se relaciona significativamente con el rendimiento académico: ámbito familiar, ya que la innovación institucional, la investigación inter, multi y transdisciplinaria, el conocimiento e innovación y la sociedad del conocimiento de la educación universitaria permite que las universidades puedan contar con el compromiso familiar y llevar un eficiente control educacional de los procesos educativos de sus hijos. El coeficiente de correlación de $r$ Pearson es de 0,38 a un nivel de significancia de 0,05 .

Cuando se realizó la confiabilidad de los instrumentos de investigación, por el método de Alfa de Cronbach, la dimensión: la innovación en la educación superior de la variable X: La Innovación Educativa y la dimensión: el ámbito familiar de la variable Y: El Rendimiento Académico tuvieron mayores coeficientes de confiabilidad de 0,70 y 0,72 respectivamente. Así mismo, en la prueba de hipótesis ambas dimensiones correlacionaban. El coeficiente de correlación de $\mathrm{r}$ Pearson es de 0,38 a un nivel de significancia de 0,05 .

\section{Literatura citada}

García, R. 2004. En su investigación Innovación, cultura $y$ poder en las instituciones educativas. Centro de Investigación y Documentación Educativa. CIDE España.

Marcelo, C.; Mayor, C. et al. 2010. En su investigación Innovación educativa en España desde el punto de vista de sus protagonistas. Universidad de Sevilla-España. 